\title{
Instrumentos de Avaliaçáo Funcional Específicos Para o Acidente Vascular Cerebral
}

\author{
Specific Functional Assessments For Stroke \\ Renan Guedes de Brito ${ }^{1}$, Livia Cristina Rodrigues Ferreira Lins ${ }^{1}$, Camila \\ Danielle Aragão Almeida ${ }^{1}$ Edmilson de Souza Ramos Neto ${ }^{2}$, Doralúcia \\ Pedrosa de Araújo ${ }^{3}$, Carlúcia Ithamar Fernandes Franco ${ }^{4}$
}

\begin{abstract}
RESUMO
Introdução. O Acidente Vascular Cerebral (AVC) é um sinal clínico com desenvolvimento rápido e perturbação focal da função cerebral, sendo a causa de diversas incapacidades, as quais predispóem os sobreviventes a um padrão de vida sedentário com limitaçôes individuais para as atividades de vida diária. Estes déficits devem ser avaliados de maneira minuciosa, fazendo-se necessário a utilização de instrumentos confiáveis e validados. Objetivo. Pesquisar escalas específicas para o AVC referenciadas na literatura mundial. Método: Foi realizada uma revisão da literatura nas bases de dados ele $\neg$ trônicas da MEDLINE, LILACS e SCIELO, referentes ao período entre 1974-2009. Resultados. Foram selecionados 32 artigos, dois periódicos que tinham em seu conteúdo capítulos que tratavam dos instrumentos de avaliação do AVC e duas dissertaçóes. Foram descritas 8 escalas: Escala de Rankin modificada, Escala de Avaliação Motora para AVC, Escala Escandinava de AVC, Escala de Avaliação Motora de Rivermead, Escala Específica de Qualidade de Vida no AVC, Escala de Braço de Frenchay, Escala do National Institute of Health para Avaliação sequencial na fase aguda do AVC e Escala de Controle de Tranco. Conclusóes. As escalas de avaliação específicas para o AVC são mundialmente conhecidas, confiáveis e validadas, sendo importantes na prática clínica do fisioterapeuta.
\end{abstract}

Unitermos. AVC, Incapacidade, Avaliação.

Citaçáo. Brito RG, Lins LCRF, Almeida CDA, Ramos Neto ES, Araújo DP, Franco CIF. Instrumentos de Avaliação Funcional Específicos Para o Acidente Vascular Cerebral.

Trabalho realizado em Campina Grande, Paraíba, Brasil.

1.Fisioterapeuta, Mestre em Ciências da Saúde pela Universidade Federal de Sergipe, Aracaju-SE, Brasil.

2.Fisioterapeuta, Mestre em Biotecnologia pela Universidade Federal do Piauí, Parnaíba-PI, Brasil.

3.Fisioterapeuta, Doutora em Ciências da Saúde pela Universidade de Brasília. Professora do Departamento de Fisioterapia da Universidade Estadual da Paraíba, Campina Grande-PB, Brasil.

4.Fisioterapeuta, Doutora em Farmacologia pela Universidade Federal da Paraíba. Professora do Departamento de Fisioterapia da Universidade Estadual da Paraíba, Campina Grande-PB, Brasil.

\begin{abstract}
Introduction. Stroke is a clinical sign with rapid and focal disruption of brain function and over 24 hours, and the leading cause of disability. The stroke's functional consequences usually predispose the survivors to a pattern of sedentary lifestyle with limited individual activities of daily living. These deficits should be evaluated in detail, being necessary to use reliable and validated instruments. Objective. Present specific scales for stroke in the referenced literature. Method. It was performed a literature review in the electronic databases MEDLINE, LILACS and SCIELO, in the period between 1974-2009. Results. It was selected 32 articles, two books that contented chapters with tools for stroke assessment and two essays. It was described eight scales: Modified Rankin Scale, Motor Assessment Scale for Stroke, Scandinavian Stroke Scale, Rivermead Motor Assessment Scale, Specific Quality of Life Scale for Scale, Frenchay Arm Scale, The National Institutes of Health Scale for sequential evaluation for stroke's acute phase and Trunk Control Test. Conclusions. The specific rating scales for stroke are known worldwide, reliable and validated, and are important in the physiotherapist's clinical practice.
\end{abstract}

Keywords. Stroke, Functionality, Measurement.

Citation. Brito RG, Lins LCRF, Almeida CDA, Ramos Neto ES, Araújo DP, Franco CIF. Specific Functional Assessments For Stroke.
Endereço para correspondência: Carlúcia IF Franco Avenida Marieta Leite, 64, Cond. Ilha Bella, Bl. Martinica, Apto. 304, Bairro Grageru. CEP 49027-190, Aracaju-SE, Brasil. Tel.: (79) 9131-6064 E-mail: guedes_renan@hotmail.com 


\section{INTRODUÇÃO}

Segundo a Organização Mundial da Saúde (OMS), o Acidente Vascular Cerebral (AVC) pode ser compreendido como uma síndrome clínica, na qual há um desenvolvimento rápido de sinais clínicos decorrentes de distúrbios focais ou globais da funçâo cerebral, com possível origem vascular e com mais de 24 horas de duração ${ }^{1}$. A causa do AVC baseia-se em dois aspectos: à uma interrupçáo do fluxo sanguíneo para o cérebro pela obstrução de uma artéria (origem isquêmica), em $80 \%$ dos casos; ou ruptura de vasos sanguíneos cerebrais (origem hemorrágica). A hipertensão arterial constitui-se um fator de risco preditivo para o AVC. Desordens cardíacas são consideradas o segundo fator de risco mais importante ${ }^{2,3}$.

O AVC é considerado, nos últimos, como um dos maiores problemas de saúde pública. É a terceira causa de morte mundial e no cenário brasileiro corresponde a primeira causa de óbito. No Brasil, a doença afeta entre 156 a 168 em cada 100.000 pessoas $^{4}$. Após os 55 anos, a incidência do AVC dobra a cada década de vida, sendo os homens os mais acometidos, cerca de $19 \%$ a mais que as mulheres. Entre 65 e 74 anos a incidência é de 14,4/1000 habitantes; entre 75 e 84 anos é de 24,6/1000; pessoas com idade superior a 85 anos, a incidência sobe para 27/1000 habitantes ${ }^{3}$.

O AVC é a primeira causa de incapacitação funcional no mundo ocidental, devido às sequelas e déficits neurológicos que ocasiona ao paciente. As sequelas são variáveis e podem ser sensitivas, motoras e/ou cognitivas, gerando prejuízos das atividades funcionais, limitação das atividades de vida diária, possível presença de déficits de linguagem, depressão e restriçóes para o convívio social ${ }^{3}$.

A fisioterapia é amplamente usada no processo de reabilitação dos indivíduos acometidos por AVC, tendo como objetivos melhorar a mobilidade funcional, a força muscular, o equilíbrio e qualidade de vida dos pacientes. Várias escalas podem ser utilizadas para avaliar o comprometimento sensório-motor após um AVC, na prática da reabilitação e em pesquisas para diagnósticos e prognósticos, bem como para mensuração da eficácia do tratamento. No entanto, as escalas que avaliam especificamente o AVC, tanto no que se refere a função motora quanto a sensitiva e cognitiva, ainda são pouco difundidas na literatura e pouco utilizadas pelos fisioterapeutas ${ }^{5,6}$.
Visando demonstrar a relevância do desenvolvimento de estudos que utilizem escalas específicas para o AVC, levando em consideração suas diversas peculiaridades clínicas, e facilitar a difusão das mesmas no meio científico e sua aplicaçâo no âmbito da Fisioterapia, o objetivo desse estudo foi apresentar, através de uma revisão da literatura, as escalas específicas para avaliar as alteraçôes motoras, sensitivas e cognitivas decorrentes do AVC referenciadas na literatura mundial, discutindo sua validade e aplicabilidade na conduta fisioterapêutica.

\section{MÉTODO}

Foram pesquisados artigos nas bases de dados eletrônicas da MEDLINE, LILACS e SCIELO, referentes ao período entre 1974-2009. As publicações incluídas nesta pesquisa foram do tipo revisóes, estudos transversais, longitudinais e ensaios clínicos, nos idiomas português, inglês e espanhol. Os descritores utilizados no levantamento foram: Acidente Vas-cular Cerebral, Qualidade de Vida, Mensuração, Incapacidade e Escalas (Stroke, Quality of Life, Measurement, Disability, Scale). Foram excluídos os artigos que descreviam tratamentos de reabilitação do AVC sem a utilização de instrumentos específicos, bem como, os que não possuíam rigor metodológico que permitisse reprodução do estudo, sendo selecionados 32 artigos, sendo estes estudos clínicos e de validação de instrumentos. Foram utilizados, ainda, dois periódicos que tinham em seu conteúdo capítulos que tratavam dos instrumentos de avaliação do AVC e duas dissertaçôes que abordavam o tema.

\section{Principais Escalas Para Avaliaçáo do Acidente Vascular Cerebral}

Foram selecionadas 8 escalas que mensuram de forma específica o grau de incapacidade funcional, incluindo os seguintes aspectos: mobilidade, equilíbrio, controle de tronco, tônus, marcha, função do membro superior, destreza manual, atividades da vida diária e qualidade de vida. Os instrumentos descritos são a Escala de Rankin modificada, Escala de Avaliação Motora para AVC, Escala Escandinava de AVC, Escala de Avaliação Motora de Rivermead, Escala Específica de Qualidade de Vida no AVC (SS - QoL), Escala de Braço de Frenchay, Escala do National Institute of Health para Avaliação sequencial 
na fase aguda do AVC e Escala de Controle de Tranco (TCT). Tais escalas podem ser aplicadas por qualquer profissional da área da saúde com treinamento prévio.

\section{Escala de Rankin de Evoluçáo Funcional após AVC}

A Escala de Rankin foi desenvolvida em 1957 por J. Rankin, com o objetivo de mensurar o grau de incapacidade e dependência nas atividades da vida diária em pacientes acometidos por AVC. A escala original é dividida em seis graus, onde o grau zero corresponde aos indivíduos sem sintomas residuais ou incapacidade e o grau cinco aos indivíduos com incapacidade grave, restrito ao leito ou à cadeira, geralmente incontinente. Posteriormente, a escala foi modificada para acréscimo do grau seis, que corresponde à morte. A escala é de rápida e fácil aplicação e apresenta uma confiança interavaliação substancial, o que indica uma confiabilidade clinicamente satisfatória, não sendo relatado dificuldades para sua aplicação ${ }^{7,8}$.

\section{Escala de Avaliaçáo Motora (EAM) para AVC}

Desenvolvida por Janet Carr em 1985, possui o objetivo de testar as intervençóes realizadas nos indivíduos acometidos por AVC. Originalmente, a escala possui 9 itens, são eles: rolar de decúbito dorsal para decúbito lateral sobre o lado bom, passar de decúbito dorsal para sentado à beira do leito, equilíbrio sentado, passar de sentado para em pé, marcha, função de membro superior, movimentos das mãos, atividades finas das mão e tônus muscular. Em 1998, a escala foi modificada, omitindo-se o item tônus muscular, o qual é pouco confiável. Cada item é pontuado de zero a seis. A escala é de rápida aplicação (10 a 15 minutos), tem confiabilidade testagem-retestagem e de interavaliação, sendo um instrumento validado. A EAM mostrou-se ser útil tanto para prática clínica quanto para utilização em pesquisas. Sugere-se a realizaçáo de treinamento antes de se iniciar a utilização dessa escala, objetivando sanar possíveis dúvidas que possam surgir, uma vez que algumas dificuldades surgiram durante a validação da escala ${ }^{9,10}$.

\section{Escala Escandinava de AVC}

Desenvolvida em 1985 pela Scandinavian Stroke Study Group, foi criada, primeiramente, para ser utilizada em uma pesquisa específica de tal grupo, sendo, poste- riormente, ajustada para utilização por não-neurologistas, tendo o objetivo de avaliar o quadro neurológico, assim como o déficit motor provocado pelo AVC. A escala foca itens de fácil acesso e de grande relevância para o paciente, são eles: consciência, orientação, linguagem/discurso, movimentos oculares, paralisia facial, marcha, braço (força/elevação), mão (força/movimentos) e perna (força/ movimentos), omitindo-se itens como sensibilidade, disartria, reflexos superficiais e profundos. A escala possui uma pontuação mínima igual a zero, o que corresponde a grave comprometimento neurológico, e uma pontuação máxima igual a cinquenta e oito, equivale a função neurológica normal. A Escala Escandinava de AVC é de rápida e fácil aplicação, tem confiabilidade interavaliação, sendo um instrumento validado. Nenhuma dificuldade de aplicação foi observada durante o processo de validação ${ }^{11}$.

\section{Escala de Avaliação Motora de Rivermead}

A escala de avaliação motora de Rivermead, medida desenvolvida em 1979 por N. Lincoln e D. Leadbitter, é uma mensuração de "função motora" pós-AVC, que visa quantificar a recuperação motora no AVC usando um modelo cumulativo conhecido como escala de Guttman, com base no pressuposto de que pacientes que sofreram um AVC seguem um padrão consistente de recuperação, sendo muito utilizada pela fisioterapia. Constitui-se de três categorias: função grosseira, a qual inclui movimentos funcionais como caminhar, sentado para de pé, virar-se e sentar na beira da cama; controle de movimento de membros inferiores e tronco e controle e funcionalidade dos membros superiores. Cada item é pontuado zero ou um, realiza ou não realiza, respectivamente, sendo que os itens seguem uma ordem de dificuldade, podendo atingir uma pontuação máxima de 38 pontos. A aplicação da escala é rápida, fácil, não sendo relatados dificuldades no processo de aplicação ${ }^{10,12}$.

\section{Escala de Braço Frenchay}

A Escala de Braço de Frenchay consiste na realização de cinco atividades básicas com o membro superior afetado pelo AVC, são elas: estabilizar uma régua enquanto faz uma linha com o lápis na outra mão; pegar um cilindro, segura-lo a aproximadamente $15 \mathrm{~cm}$ da extremidade da mesa, levantá-lo aproximadamente $30 \mathrm{~cm} \mathrm{e}$ 
coloca-lo na mesma posição sem deixar cair; levantar um copo com água pela metade, posiciona-lo 15 a $30 \mathrm{~cm} \mathrm{da}$ extremidade da mesa, beber um pouco e recoloca-lo no lugar sem derramar a água; remover e recolocar no lugar um prendedor de roupa de varal e pentear o cabelo. Em cada atividade, o paciente pode obter o escore 1 (realizou com sucesso) ou 0 (insucesso), podendo obter uma soma geral de 0 (incapacidade completa) a 5 (ausência de incapacidade). O teste é de rápida e fácil aplicação, menos que 3 minutos, apresentando uma confiança interavaliação substancial, o que indica uma confiabilidade clinicamente satisfatória ${ }^{12}$.

\section{Escala Específica de Qualidade de Vida no AVC (SS- -QoL)}

Desenvolvida em 1999 a partir de entrevistas com hemiplégicos que identificaram as áreas mais afetadas pelo AVC, é uma escala específica de avaliação da qualidade de vida em pacientes após AVC. Em sua forma original possui 49 itens em 12 domínios (energia, papel familiar, linguagem, mobilidade, humor, personalidade, autocuidado, papel social, raciocínio, função de membro superior, visão e trabalho/produtividade) e para cada item existem escores de até 5 pontos ${ }^{4,13}$. O instrumento é de simples aplicação e compreensão dos itens, bem como na interpretação dos dados obtidos, porém sua aplicação leva um tempo considerável, cerca de 40 minutos $^{13}$. Por ser uma escala específica, mostra-se válida para acessar alteraçóes significativas da qualidade de vida dentro das dimensôes dos sintomas observados, sendo uma escala direcionada às particularidades e domínios mais afetados nos pacientes após $\mathrm{AVC}^{4}$.

\section{Escala do National Institute of Health para Avaliaçáo sequencial na fase aguda do AVC (NIHSS)}

A escala do National Institutes of Health Stroke (NIHSS), desenvolvida pelos Institutos da Saúde norte-americano, constitui-se em um dos instrumentos de avaliação mais utilizados internacionalmente, sendo um indicador do tamanho e gravidade da lesão, bem como do prognóstico de pacientes acometidos por $\mathrm{AVC}^{14}$. Esta escala é composta por 11 itens, sendo cada um deles mensurável de acordo com o déficit apresentado. Aproximadamente 60 a $70 \%$ dos pacientes com AVC isquêmico agudo com pontuação $<10$ apresentarão prognóstico favorável após um ano, se comparado com 4 a 16\% daqueles que mostrarem escores $>20$. Ainda de acordo com estes autores, a pontuação na NIHSS ajuda a identificar aqueles pacientes com grandes riscos de hemorragia craniana associada a tratamento trombolítico, sendo de fácil e rápida aplicação ${ }^{15}$.

\section{Escala de Controle de Tronco (TCT)}

Consiste em um instrumento utilizado para avaliar o prejuízo motor em pacientes acometidos por AVC. A TCT, além de avaliar a manutenção da posição sentada, examina a habilidade do indivíduo rolar da posição supina para o lado afetado e sã, bem como a transferência da posição supina para sentada ${ }^{16}$. Em cada teste a pontuação varia de 0 a 25 , sendo que o 0 corresponde a incapacidade da execução da atividade sem ajuda, o 12 a realização da atividade sem ajuda muscular e o 25 a execução da tarefa normalmente ${ }^{17}$.

\section{DISCUSSÃO}

A capacidade funcional surge como um novo paradigma de saúde, particularmente, um valor ideal para que se possa viver independente, sendo esta a capacidade do indivíduo realizar atividades físicas e mentais necessárias para manutenção de suas atividades básicas e instrumentais $^{18}$. A perda dessa capacidade está associada à predição de fragilidade, dependência, institucionalização, risco aumentado de quedas, morte e problemas de mobilidade, trazendo complicaçóes ao longo do tempo e gerando cuidados de longa permanência e alto custo ${ }^{19}$.

As sequelas provenientes do AVC implicam em graus variados de dependência, principalmente, no primeiro ano após o AVC. Cerca de 30 a 40\% dos sobreviventes tornam-se impossibilitados de voltarem ao trabalho e requerem algum tipo de auxílio no desempenho de atividades cotidianas básicas ${ }^{20}$; e $10 \%$ ficam incapacitados de viver na comunidade, necessitando de cuidados de terceiros e demência, em geral devido a uma combinação de falta de apoio social e incapacidade grave ${ }^{21}$.

A ocorrência do AVC frequentemente traz consequências negativas para o sobrevivente, como aumento da dependência de outras pessoas para as atividades da vida diária, alterações do humor e uma ruptura na interação 
social com os amigos e os membros da própria família, o que pode resultar em um importante rebaixamento da qualidade de vida destes indivíduos ${ }^{22}$.

A reabilitação do paciente com sequela de AVC é na maioria das vezes um grande desafio. Os esforços para minimizar o impacto e para aumentar a recuperação funcional após AVC têm sido ponto importante para os profissionais de reabilitação. Para tanto, faz-se necessário avaliaçóes acuradas, a fim de determinar e documentar os resultados dos programas de reabilitação ${ }^{23}$.

As informações, coletadas nas avaliações, são fundamentais para o planejamento, evolução e modificação do programa de treinamento, sendo um importante meio de estabelecer o ingresso do paciente a reabilitação, melhor prática e possibilidade de realizar mudança de práticas à medida que novos métodos de intervenção são desenvolvidos, fazendo com que a fisioterapia cumpra seu papel principal: proporcionar o retorno do indivíduo às suas atividades de lazer, domésticas e de trabalho, e à sua função independente na comunidade ${ }^{12}$.

A Escala de Rankin é utilizada para determinar o nível de comprometimento neurológico, com níveis de evidência de confiabilidade clinicamente aceitáveis e de boa aplicabilidade ${ }^{7}$, corroborando com um estudo realizado por Wilson et al. ${ }^{24}$, que obteve um índice de confiabilidade de 0,45 .

A EAM tem sido extensivamente utilizada em pesquisa e na clínica, incluindo aspectos qualitativos e quantitativos de tarefas funcionais. A escala possui alta confiabilidade e boa aceitação pelos fisioterapeutas ${ }^{25}$. Porém, devido à grande quantidade de dúvidas na pontuação dos pacientes, sugere-se treinamento prévio à utilização clínica dessa escala, corroborando o artigo pioneiro que afirma que a EAM é confiável e validada, recomendando que os fisioterapeutas realizem a aplicação da escala em no mínimo 6 pacientes como forma de treinamento, antes de incluí-la na prática clínica?.

A Escala Escandinava para AVC é mundialmente utilizada e apresenta uma grande capacidade de prognóstico, sendo capaz de mensurar o risco de morte, a incapacidade, o tempo de permanência no hospital e as possibilidades de institucionalização, sendo, sua utilização viável na prática clínica ${ }^{26}$, corroborando o artigo pioneiro da Scandinavian Stroke Study Group ${ }^{11}$.
A confiabilidade inter, intra-avaliador e intraobservadores da Escala de Avaliação Motora de Rivermead foi avaliada e chegou-se a conclusão que esta apresenta uma boa confiabilidade ${ }^{27,28}$, sendo confiável para aplicação clínica $^{10}$.

A Escala de Braço de Frenchay possui uma boa confiabilidade, sendo capaz de avaliar o controle proximal do membro superior e a destreza manual, porém, a escala não consegue mensurar pequenas alteraçóes da função do membro superior ${ }^{29,30}$.

A SS-QoL aprensenta uma boa avaliação das atividades psicométricas, sendo validada, confiável e sensível $^{13,31}$. A escala possui boa validade de conteúdo, abrangendo frequentemente áreas negligenciadas por outros questionários como a linguagem e a cogniçãa ${ }^{31}$. A versão em português da escala mostrou-se um instrumento clinicamente útil, demonstrando boa confiabilidade das medidas, estabilidade nas respostas e permitindo a discriminação de indivíduos e itens em diferentes níveis de qualidade de vida ${ }^{32}$.

A NIHSS é a medida padrão para avaliar a função neurológica dos pacientes, por ser uma escala validada, confiável e, principalmente, por auxiliar no seguimento neurológico e na decisão terapêutica durante o período de hospitalização dos indivíduos acometidos por $\mathrm{AVC}^{33,34}$.

A TCT é uma escala que pode ser utilizada para avaliar o prognóstico funcional dos indivíduos pós-AVC, sendo uma escala validada e confiável ${ }^{35}$. Tal escala vem sendo amplamente utilizada, uma vez que a avaliação clínica do controle de tronco constitui-se uma ferramenta importante para o prognóstico das habilidades funcionais de pacientes $^{36}$.

O uso de escalas é de suma importância na prática clínica de qualquer profissional, porém, a escolha de um instrumento de avaliação depende diretamente das características do serviço onde o mesmo será utilizado, variáveis como tempo de administraçáo, custo de aplicação, treinamento dos profissionais e a disponibilidade de manual de instruçóes influenciam nesta escolha ${ }^{10}$.

\section{CONCLUSÃO}

As escalas específicas para o AVC são mundialmente conhecidas, confiáveis e validadas, sendo importantes na prática clínica do fisioterapeuta, uma vez que a 
mensuração acurada do estado funcional do paciente é de grande relevância, pois é a partir dela que se pode traçar um programa fisioterapêutico adequado às especificidades de cada indivíduo.

\section{REFERÊNCIAS}

1.Iwabe C, Diaz MAR, Barudy DP. Análise Cinemática da Marcha em Indivíduos com Acidente Vascular Encefálico. Rev Neurocienc 2008;16(4):292-6. 2.Benvegnu AB, Gomes LA, Souza CT, Cuadros TBB, Pavão LW, Ávila SN. Avaliaçấo da Medida de Independência Funcional de indivíduos com seqüela de Acidente Vascular Encefálico (AVE). Ciênc Saúde Colet 2008;1(2):71-77. 3.Polese JC, Tonial A, Fung FK, Mazuco R, Oliveira SG, Schuster RC. Avaliação da Funcionalidade de Indivíduos Acometidos por Acidente Vascular Encefálico. Rev Neurocienc 2008;16(3):175-8.

4. Oliveira MR, Orsini M. Escalas de Avaliação de qualidade de vida em pacientes brasileiros após Acidente Vascular Encefálico. Rev Neurocienc 2008; 17(3):255-62.

5.Bullock EA, Lupton D. Later stages of rehabilitation in hemiplegia. Physiotherapy 1974;60(12):370-4.

6.Rodrigues JE, Sá MS, Alouche SG. Perfil dos pacientes acometidos por AVE tratados na clínica escola de fisioterapia da UMESP. Rev Neurocienc 2004;12(3):117-22.

7.Caneda MAG, Fernandes JG, Almeida AG, Mugnol FE. Confiabilidade de escalas de comprometimento neurológico em pacientes com Acidente Vascular Cerebral. Arq Neuropsiquiatr 2006;64(3-A):690-7.

http://dx.doi.org/10.1590/S0004-282X2006000400034

8.André C. Manual de AVC. 2 ed. Rio de Janeiro: Revinter, 2006, 45p.

9.Carr JH, Shepherd RB, Nordholm L, Lynne D. Investigation of a new motor assessment scale for stroke patients. Phys Ther 1985;65(2):175-80.

10.Carvalho TB, Relvas PCA, Rosa SF. Instrumentos de avaliação da função motora para indivíduos com lesão encefálica adquirida. Rev Neurocienc 2008;16(2):137-43.

11.Scandinavian Stroke Study Group. Multicenter Trial of Hemodilution in Ischemic Stroke Background and Study Protocol. Stroke 1985;16(5):885-90. http://dx.doi.org/10.1161/01.STR.16.5.885

12.Carr J, Sheperd R. Reabilitaçáo neurológica: otimizando o desempenho motor. $1^{\text {a }}$ ed. São Paulo: Manole, 2002, 115p.

13.Williams LS, Weinberger M, Harris LE, Clark DO, Biller J. Development of a Stroke-Specific Quality of Life Scale. Stroke 1999;30(7):1362-9.

http://dx.doi.org/10.1161/01.STR.30.7.1362

14.Rocha SIM. Doença Cerebrovascular Isquêmica Aguda: Avaliação de Protocolo de Trombólise (Dissertação). Covilhă: Universidade da Beira Interior, 2008, 112p.

15.Adams Jr HP, Adams RJ, Brott T, del Zoppo GJ, Furlan A, Goldstein LB, et al. Guidelines for the Early Management of Patients With Ischemic Stroke - A Scientific Statement From the Stroke Council of the American Stroke Association. Stroke 2003;34:1056-83.

http://dx.doi.org/10.1161/01.STR.0000064841.47697.22

16.Franchignoni FP, Tesio L, Ricupero C, Martino MT. Trunk Control Test as an Early Predictor of Stroke Rehabilitation Outcome. Stroke 1997;28:1382-5. http://dx.doi.org/10.1161/01.STR.28.7.1382
17.Colin C, Wade D. Assessing motor impairment after stroke: a pilotreliability study. J Neurol Neurosurg Psychiatry 1990;53:576-9.

http://dx.doi.org/10.1136/jnnp.53.7.576

18.Ramos LR. Fatores determinantes do envelhecimento saudável em idosos residentes em centro urbano: Projeto Epidoso, São Paulo 2003, p.214-57.

19.Cordeiro RC, Dias RC, Dias JMD, Perracini M, Ramos LR. Concordância entre observadores de um protocolo de avaliação fisioterapêutica em idosas institucionalizadas. Rev Bras Fisioter 2002;9:69-77.

20.Falcão IV, Carvalho EMF, Barreto KML, Lessa FJD, Leite VMM. Acidente Vascular Cerebral precoce: implicaçóes para adultos em idade produtiva atendidos pelo Sistema Único de Saúde. Rev Bras Saúde Mater Infant 2004;4(1):95-102.

http://dx.doi.org/10.1590/S1519-38292004000100009

21.Pereira S. Acidente Vascular Cerebral: hospitalização, mortalidade e prognóstico. Acta Med Port 2004;17:187-92.

22.Cordini KL, Oda EY, Furnaletto LM. Qualidade de vida de pacientes com história prévia de acidente vascular cerebral: observação de casos. Rev Bras Psiquiatr 2005;54(4):312-31.

23.Cacho EWA, Melo FRLV, Oliveira R. Avaliçáo da recuperação motora de pacientes hemiplégicos através do protocolo de desempenho físico Fulg-Meyer. Rev Neurocienc 2004;12(2):94-102.

24.Wilson JT, Hareendran A, Grant M, Baird T, Schulz UG, Muir KW, Bone I. Improving the assessment of outcome in stroke: use of a structured interview to assign grades on the modified Rankin Scale. Stroke 2002;33:2243-6.

http://dx.doi.org/10.1161/01.STR.0000027437.22450.BD

25.Conte ALF, Ferrari PP, Carvalho TB, Relvas PCA, Neves RCM, Rosa SF. Confiabilidade, compreensão e aceitação da versão em português da Motor Assessment Scale em pacientes com acidente vascular encefálico. Rev Bras Fisioter 2009; 13(5): 405-11.

http://dx.doi.org/10.1590/S1413-35552009005000056

26.Sprigg NG, Gray LJ, Bath PMW, Lindenstrøm E, Boysen G, De Deyn PP, et al. Stroke severity, early recovery and outcome are each related with clinical classification of stroke: data from the Tinzaparin in Acute Ischemic Stroke Trial (TAIST). J Neurol Sci 2007; 254 (1-2):54-9.

http://dx.doi.org/10.1016/j.jns.2006.12.016

27.Lincoln N, Leadbitter D. Assessment of Motor Function in Stroke Patients. Physiotherapy 1979;65(2):48-51.

28.Collen FM, Wade DT, Bradshaw CM. Mobility after stroke: reliability of measures of impairment and disability. Int Disabil Stud 1990;12(1):6-9.

http://dx.doi.org/10.3109/03790799009166594

29.Hsieh C, Hsueh IP, Chiang FM, Lin PH. Inter-rater reliability and validity of the Action Research arm test in stroke patients. Age Ageing 1998;27:10713.

http://dx.doi.org/10.1093/ageing/27.2.107

30.Heller C, Wade DT, Wood VA, Sunderland A, Hewer RL, Ward E. Arm function after stroke: measurement and recovery over the first three months. J Neurol Neurosurg Psychiatry 1987;50:714-9.

http://dx.doi.org/10.1136/jnnp.50.6.714

31.Duncan PW, Jorgensen HS, Wade DT. Outcome measures in acute stroke trials. Stroke 2000;31:1429-38.

http://dx.doi.org/10.1161/01.STR.31.6.1429

32.Lima, RCM. Adaptaçấo Transcultural Do Stroke Specific Quality Of Life - Ssqol: Um instrumento específico para avaliar a qualidade de vida de hemiplégicos (Dissertação). Belo Horizonte: Universidade Federal de Minas Gerais, 2006, 77p.

33.Hacke W, Schwab S, Horn M, Spranger M, De Georgia M, von Kummer R. Malignant middle cerebral artery infarction: clinical course and prognostic 
signs. Arch Neurol 1996;53:309-15.

http://dx.doi.org/10.1001/archneur.1996.00550040037012

34.Goldstein LB, Samsa GP. Reliability of the National Institutes of Health Stroke Scale: extension to non-neurologists in the context of a clinical trial. Stroke 1997;28:307-10.

http://dx.doi.org/10.1161/01.STR.28.2.307
35.Duarte E, Marco E, Muniesa JM, Belmonte R, Diaz P, Tejero M, Escalada F. Trunk Control Test as a functional predictor in stroke patients. J Rehabil Med 2002;34:267-72.

http://dx.doi.org/10.1080/165019702760390356

36.Aguiar PT, Rocha TN, Oliveira ES. Escalas de controle de tronco como prognóstico funcional em pacientes após acidente vascular. Acta Fisiátrica 2008;15(3):160-4. 